\title{
Color dipole model bounds with the gluon-gluon recombination correction
}

\author{
G. R. Boroun $\oplus^{*}$ and B. Rezaei $\odot^{\dagger}$ \\ Department of Physics, Razi University, Kermanshah 67149, Iran
}

(Received 31 March 2021; accepted 1 June 2021; published 23 June 2021)

\begin{abstract}
We present nonlinear (NL) and higher-twist (HT) corrections to the color dipole model bounds at low values of $x$ and $Q^{2}$ using the parametrization method. Consistency between the bounds at this region describe that a transition from the linear to the nonlinear behavior is dependence on the behavior of the gluon distribution function. The parameters in the color dipole model are comparable with the color dipole bounds at low values of $Q^{2}$. Consequently, the obtained reduced cross sections at low- and moderate- $Q^{2}$ values due to the NL $+\mathrm{HT}$ effects show good agreement with the H1 Collaboration data.
\end{abstract}

DOI: 10.1103/PhysRevC.103.065202

\section{INTRODUCTION}

The starting points on the color dipole model were given by Sakurai and Schildknecht in 1972 [1] and has been expanded so far by some authors in Refs. [2-4]. The modern picture of the deep inelastic scattering (DIS) at low $x$ is described as the color dipole picture (CDP). In this picture the virtual photon fluctuates into the $q \bar{q}$ pair which this pair interaction with the gluon field in the nucleon as a gauge-invariant color-dipole interaction. Due to the interaction of the gluon fields with the $q \bar{q}$ dipole, the dipole cross section $\sigma_{(q \bar{q})_{L, T}^{J=1} p}$ is described at the color transparency and saturation limits. The $W^{2}$-dependent scale $\Lambda_{\text {sat }}^{2}\left(W^{2}\right)$ separates the two regions. The color transparency of the dipole cross section according to the region of $Q^{2} \gg \Lambda_{\text {sat }}^{2}\left(W^{2}\right)$ and the saturation according to the region of $Q^{2} \ll \Lambda_{\text {sat }}^{2}\left(W^{2}\right)$, respectively. Indeed the $\left(Q^{2}, W^{2}\right)$ plane of the CDP indicates that the line $\eta\left(W^{2}, Q^{2}\right)=1$ subdivides the $\left(Q^{2}, W^{2}\right)$ plane into the saturation region of $\eta\left(W^{2}, Q^{2}\right)<1$ and the color transparency region of $\eta\left(W^{2}, Q^{2}\right)>1 . \eta\left(W^{2}, Q^{2}\right)$ denotes the low- $x$ scaling variable $\eta\left(W^{2}, Q^{2}\right)=\frac{Q^{2}+m_{0}^{2}}{\Lambda_{\text {sat }}^{2}\left(W^{2}\right)}$ where $\Lambda_{\text {sat }}^{2}\left(W^{2}\right)$ is the saturation scale and $m_{0}^{2} \simeq 0.15 \mathrm{GeV}^{2}$. At low- $x$ scaling, the total photoabsorption cross-section $\sigma_{\gamma^{*} p}\left(W^{2}, Q^{2}\right)=$ $\sigma_{\gamma^{*} p}\left[\eta\left(W^{2}, Q^{2}\right)\right]$ is described as $\ln \left[1 / \eta\left(W^{2}, Q^{2}\right)\right]$ for $\eta\left(W^{2}, Q^{2}\right)<1$ and as $1 / \eta\left(W^{2}, Q^{2}\right)$ for $\eta\left(W^{2}, Q^{2}\right) \gg 1$. At large $Q^{2} \gg \Lambda_{\text {sat }}^{2}\left(W^{2}\right)$, the longitudinal-to-transverse ratio of the photoabsorption cross-sections $\sigma_{\gamma_{L}^{*} p}\left(W^{2}, Q^{2}\right)$ and $\sigma_{\gamma_{T}^{*} p}\left(W^{2}, Q^{2}\right)$ reads as

$$
R\left(W^{2}, Q^{2}\right)=\frac{\sigma_{\gamma_{L}^{*} p}\left(W^{2}, Q^{2}\right)}{\sigma_{\gamma_{T}^{*} p}\left(W^{2}, Q^{2}\right)}=\frac{1}{2 \rho} .
$$

\footnotetext{
*grboroun@gmail.com; boroun@razi.ac.ir

†brezaei@razi.ac.ir
}

Published by the American Physical Society under the terms of the Creative Commons Attribution 4.0 International license. Further distribution of this work must maintain attribution to the author(s) and the published article's title, journal citation, and DOI. Funded by $S C O A P^{3}$.
In terms of the proton structure functions, the ratio of the structure functions becomes

$$
\frac{F_{L}\left(W^{2}, Q^{2}\right)}{F_{2}\left(W^{2}, Q^{2}\right)}=\frac{1}{1+2 \rho} .
$$

The parameter $\rho$ is associated with the enhanced transverse size of $q \bar{q}$ fluctuations in the color dipole model (CDM). This parameter is originating from transverse $\gamma_{T}^{*} \rightarrow q \bar{q}$ and longitudinal $\gamma_{L}^{*} \rightarrow q \bar{q}$ photons. Indeed the $\rho$ parameter describes the ratio of the average transverse momenta $\rho=\frac{\left\langle\vec{k}_{\perp}^{2}\right\rangle_{L}}{\left\langle\vec{k}_{\perp}^{2}\right\rangle_{T}}$. It can also be related to the ratio of the effective transverse sizes of the $(q \bar{q})_{L, T}^{J=1}$ states as

$\frac{\left\langle\vec{r}_{\perp}^{2}\right\rangle_{L}}{\left\langle\vec{r}^{2}\right\rangle_{T}}=\frac{1}{\rho}$. The $\rho$ parameter is assumed to be proportional to the singlet structure and gluon distribution functions in the large- $Q^{2}$ limit [5],

$$
\rho\left(x, Q^{2}\right)=\frac{3 \pi}{8 \alpha_{s}\left(Q^{2}\right)} \frac{F_{2}^{s}\left(x, Q^{2}\right)}{G\left(x, Q^{2}\right)}-\frac{1}{2},
$$

where $F_{2}^{s}\left(x, Q^{2}\right)=x \Sigma\left(x, Q^{2}\right)$ and $G\left(x, Q^{2}\right)=x g\left(x, Q^{2}\right)$.

In this paper we want to show that the behavior of the CDM bounds at low- and moderate- $Q^{2}$ values are depends on the gluon density behavior. In this case the bounds are obtained via the nonlinear-Dokshitzer-Gribov-Lipatov-Altarelli-Parisi (DGLAP) evolution. Studies along this line not only confirm HERA investigations, but also provide crucial benchmarks for further investigations of the high-energy limit of QCD at the Electron-Ion Collider (EIC) [6] and the large Hadron Electron Collider ( $\mathrm{LHeC})[7,8]$. The kinematic extension of the $\mathrm{LHeC}$ will allow us to examine the nonlinear dynamics at low $x$. The nonlinear region is approached when the reaction is mediated by multigluon exchange. Indeed the growth of the gluon density is slowed down at very small $x$ by the gluongluon recombination process. The kinematic coverage of the neutral-current $e^{-} p$-scattering pseudodata at the $\mathrm{LHeC}$ which indicate the nonlinear dynamics are defined in the regions $x<0.01$ and $Q^{2}<700 \mathrm{GeV}^{2}[9,10]$. At small $x$ the effect of $\propto \ln (1 / x)$ terms on the linear evolution equations increases. So nonlinear interactions must be applied. Indeed we need reliable $\mathrm{LHeC}$ predictions to understand the low- $x$ physics 
[11]. Since nonlinear dynamics are known to become sizable only at small $x$, so the nonlinear contribution to the evolution equation [12] leads to an equation of the form

$$
\frac{\partial^{2} x g\left(x, Q^{2}\right)}{\partial \ln (1 / x) \partial \ln Q^{2}}=\bar{\alpha}_{s} x g\left(x, Q^{2}\right)-\frac{9}{16} \bar{\alpha}_{s}^{2} \pi^{2} \frac{\left[x g\left(x, Q^{2}\right)\right]^{2}}{\mathcal{R}^{2} Q^{2}},
$$

where $\bar{\alpha}_{s} \equiv \alpha_{s} C_{A} / \pi$ and the parameter $\mathcal{R}$ controls the strength of the nonlinearity. The second nonlinear term in (4) is responsible for gluon recombination. This term arises from perturbative QCD diagrams which couple four gluons to two gluons so that two gluon ladders recombine into a single gluon ladder. It leads to saturation of the gluon density at low $Q^{2}$ with decreasing $x$ [13]. The gluon recombination is as important as gluon splitting which in analysis some groups, such as Refs. [14,15], in next-to-leading-order (NLO) analysis considered. This implies that towards small values of $x$ and $Q^{2}$ the problem of negative gluon distribution in these groups appears. Other nonlinear equations, such as modified DGLAP [16], Balitsky-Kovchegov [17], and JalilianMarian-McLerran-Weigert-Leonidov-Kovner [18] equations have been derived and considered in the past years. Some another models, such as the impact-parameter-dependent saturation model [9], developed a dipole model for DESY HERA which incorporates the impact parameter distribution of the proton. It is a simple dipole model that incorporates key features of the physics of gluon saturation. This model for the dipole amplitude contains an eikonalized gluon distribution which satisfies DGLAP evolution whereas explicitly maintaining unitarity [19]. In Ref. [20] the nonlinear evolution equation for dipole density has been developed. The deeply inelastic scattering at very high energies in the saturation regime was considered.

The unitarity problem is discussed in Ref. [21] with respect to photoabsorption cross sections. The unitarity relation entails the nonlinearity of the observed DIS structure functions in terms of the impulse approximation parton densities. The expectation value of the interaction cross sections of the multiparton Fock states of the virtual photon over the wave functions is considered in Ref. [21]. The unitarized total crosssections $\sigma(x, \rho)$ read

$$
\sigma(x, \rho) \simeq \sigma_{0}(x, \rho) \quad \text { at } \quad \eta(x, \rho) \ll 1,
$$

where the quantity $\eta(x, \rho)$ controls the effect of the unitarization and $\rho$ is the transverse size of the $q \bar{q}$ pair. At $\eta(x, \rho) \gg 1$ the unitarization suppresses the cross section as $\sigma(x, \rho) \ll$ $\sigma_{0}(x, \rho)$ where $\sigma_{0}(x, \rho)$ is the interaction cross section for the $q \bar{q}$-color dipole of size $\rho$. The effects of the $q \bar{q} g$-Fock state is the deriving term of the triple-pomeron mass spectrum. The shadowing term in the unitarized structure function is dominated by the triple-pomeron term, which is approximately independent of the flavor and $Q^{2}$ variables. Indeed the unitarity (shadowing) correction is a nonlinear functional of the DGLAP cross section. Also the unitarity correction can be related to the cross section of the forward diffractive dissociation of the virtual photons (DDIS) $\gamma^{*}+p \rightarrow X+p$ where $X=q \bar{q}$. The conventional description of DDIS is based on the leading twist DGLAP evolution equations which characterize the QCD hard scale dependence of the diffractive parton distribution functions [22]. The effects of pomeron loops and running coupling on the cross sections for inclusive $\gamma^{*} h$ and on diffractive deep inelastic scattering are investigated in Ref. [23]. In Ref. [24] DDIS provides a basis for the definition of the Weizsäcker-Williams nuclear gluon structure function. Also the initial conditions at low- $x$ DIS off nucleons and nuclei for QCD evolution that satisfy unitarity are described. The nonlinear effects can be tested at a superior statistical accuracy attainable at the EIC.

This paper is organized as follows. In the next section the theoretical formalism is presented, including the nonlinear evolution and the color dipole parameters. In Sec. III, we present a detailed numerical analysis and our main results. We then confront these results with the CDM bounds at low values of $Q^{2}$. In the last section we summarize our main conclusions and remarks.

\section{THEORETICAL FORMALISM}

In the CDM the $\rho$ parameter is dependent on the proton structure function $F_{2}\left(x, Q^{2}\right)$ and the gluon distribution function $G\left(x, Q^{2}\right)$ as reads

$$
\rho\left(x, Q^{2}\right)=\frac{27 \pi}{20 \alpha_{s}\left(Q^{2}\right)} \frac{F_{2}\left(x, Q^{2}\right)}{G\left(x, Q^{2}\right)}-\frac{1}{2} .
$$

An analytical expression for $F_{2}\left(x, Q^{2}\right)$ has suggested which describes fairly well the available experimental data on the reduced cross section [25]. This parametrization provides reliable structure function $F_{2}\left(x, Q^{2}\right)$ according to HERA data at low $x$ in a wide range of the momentum transfer $\left(1 \mathrm{GeV}^{2}<\right.$ $Q^{2}<3000 \mathrm{GeV}^{2}$ ) as

$$
F_{2}\left(x, Q^{2}\right)=D\left(Q^{2}\right)(1-x)^{n} \sum_{m=0}^{2} A_{m}\left(Q^{2}\right) L^{m},
$$

and can be applied as well in analyses of ultra-high-energy processes with cosmic neutrinos. In a new method, the linear behavior of the gluon density in the CDM parameters is investigated in Ref. [26]. Now we consider the nonlinear behavior of the gluon density for the CDM bounds. The nonlinear effects of the gluon-gluon fusion due to the high gluon density at small $x$ is considered in Gribov-Levin-Ryskin-Mueller-Qiu (GLR-MQ) [12]. Some studies of the GLR-MQ equation in the framework of the extracting the gluon distribution function have been discussed considerably over the past years [27-33]. The GLR-MQ equation can be written in standard form [34]

$$
\begin{aligned}
\frac{\partial G\left(x, Q^{2}\right)}{\partial \ln Q^{2}}= & \left.\frac{\partial G\left(x, Q^{2}\right)}{\partial \ln Q^{2}}\right|_{\text {DGLAP }} \\
& -\frac{81}{16} \frac{\alpha_{s}\left(Q^{2}\right)}{\mathcal{R}^{2} Q^{2}} \int_{\chi}^{1} \frac{d z}{z} G^{2}\left(\frac{x}{z}, Q^{2}\right),
\end{aligned}
$$

where $\chi=\frac{x}{x_{0}}$ and $x_{0}$ is the boundary condition that the gluon distribution joins smoothly onto the linear region. The correlation length $\mathcal{R}$ determines the size of the nonlinear terms. This value depends on how the gluon ladders are coupled to the nucleon or on how the gluons are distributed within the nucleon. $\mathcal{R}$ is approximately equal to $\simeq 5 \mathrm{GeV}^{-1}$ if the gluons are populated across the proton, and it is equal to $\simeq 2 \mathrm{GeV}^{-1}$ if the gluons have hot-spot-like structures. By solving GLR-MQ 
[Eq. (7)], we obtain an expression for the nonlinear gluon distribution function [i.e., $G^{\mathrm{NL}}\left(x, Q^{2}\right)$ ] as

$$
\begin{aligned}
G^{\mathrm{NL}}\left(x, Q^{2}\right)= & G^{\mathrm{NL}}\left(x, Q_{0}^{2}\right)+G\left(x, Q^{2}\right)-G\left(x, Q_{0}^{2}\right) \\
& -\int_{Q_{0}^{2}}^{Q^{2}} \frac{81}{16} \frac{\alpha_{s}\left(Q^{2}\right)}{\mathcal{R}^{2} Q^{2}} \int_{\chi}^{1} \frac{d z}{z} G^{2}\left(\frac{x}{z}, Q^{2}\right) d \ln Q^{2} .
\end{aligned}
$$

We note that at $x \geqslant x_{0}\left(=10^{-2}\right)$ the linear and nonlinear gluon distribution behaviors are equal. At $Q_{0}^{2}$ the low-x behavior of the nonlinear gluon distribution is assumed to be [35]

$$
\begin{aligned}
G^{\mathrm{NL}}\left(x, Q_{0}^{2}\right)= & G\left(x, Q_{0}^{2}\right)\left\{1+\frac{27 \pi \alpha_{s}\left(Q_{0}^{2}\right)}{16 \mathcal{R}^{2} Q_{0}^{2}} \theta\left(x_{0}-x\right)\right. \\
& \left.\times\left[G\left(x, Q_{0}^{2}\right)-G\left(x_{0}, Q_{0}^{2}\right)\right]\right\}^{-1} .
\end{aligned}
$$

Substituting Eqs. (6) and (8) in Eq. (5) the nonlinear behavior of the $\rho$ parameter becomes

$$
\rho^{\mathrm{NL}}\left(x, Q^{2}\right)=\frac{27 \pi}{20 \alpha_{s}\left(Q^{2}\right)} \frac{F_{2}\left(x, Q^{2}\right)[\text { i.e., Eq. }(6)]}{G^{\mathrm{NL}}\left(x, Q^{2}\right)[\text { i.e., Eq. (8)] }}-\frac{1}{2} .
$$

Next we define the nonlinear behavior of the longitudinal-totransverse cross sections and the structure functions by the following forms, respectively:

$$
R^{\mathrm{NL}}\left(W^{2}, Q^{2}\right)=\frac{1}{2 \rho^{\mathrm{NL}}\left(W^{2}, Q^{2}\right)},
$$

and

$$
F_{L / 2}^{\mathrm{NL}}\left(W^{2}, Q^{2}\right) \equiv \frac{F_{L}\left(W^{2}, Q^{2}\right)}{F_{2}\left(W^{2}, Q^{2}\right)}=\frac{1}{1+2 \rho^{\mathrm{NL}}\left(W^{2}, Q^{2}\right)} .
$$

If we rewrite the reduced cross section in terms of the nonlinear behavior of the ratio of the structure functions, then, the nonlinear behavior of the reduced cross section at low $Q^{2}$ reads

$$
\begin{aligned}
& \sigma_{r}^{\mathrm{NL}}\left(W^{2}, Q^{2}\right) \\
& \quad=F_{2}\left(W^{2}, Q^{2}\right)\left[1-\frac{y^{2}}{1+(1-y)^{2}} \frac{1}{1+2 \rho^{\mathrm{NL}}\left(W^{2}, Q^{2}\right)}\right] .
\end{aligned}
$$

Here $W^{2} \simeq s y$ which the inelasticity $y$ is related to $Q^{2}, x$, and the center-of-mass energy squared $s=4 E_{e} E_{p}$ by $y=Q^{2} / s x$.

In the following we consider the deeply inelastic structure functions at low $Q^{2}$ using the higher-twist (HT) corrections in QCD. Using this effect in the parametrization of the proton structure function is expected to provide better results for the reduced cross section than the experimental data. The highertwist corrections arise from the struck proton's interaction with target remnants where reflecting confinement [36-40]. The phenomenological power correction to the structure function from the HT corrections is considered by the following form:

$$
F_{2}^{\mathrm{HT}}\left(x, Q^{2}\right)=F_{2}^{\text {parametrization }}\left(x, Q^{2}\right)\left(1+\frac{C_{\mathrm{HT}}(x)}{Q^{2}}\right),
$$

which the coefficient function $C_{\mathrm{HT}}(x)$ is determined from fit to the data. In some references [37-41] this quantity is set to be

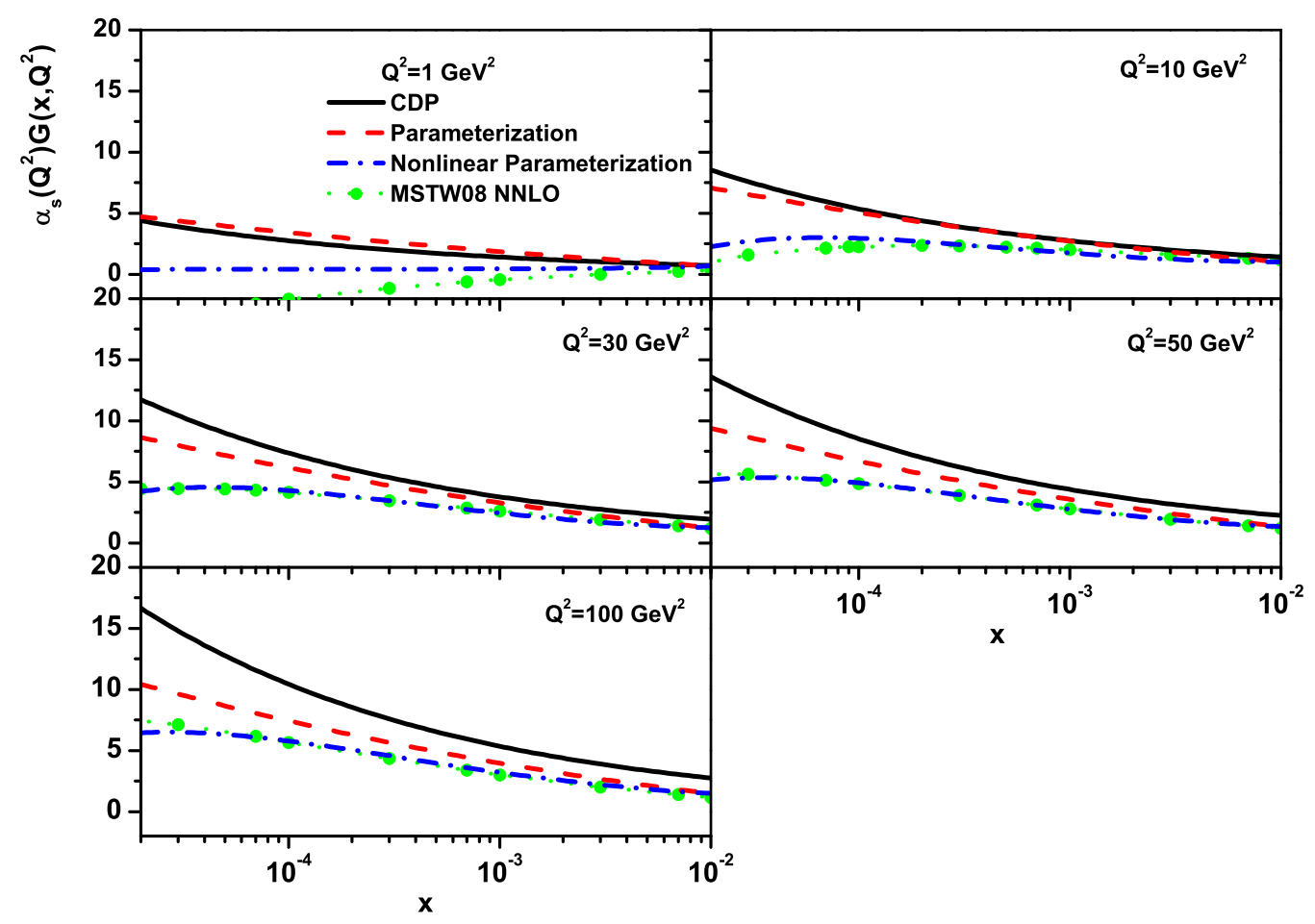

FIG. 1. The nonlinear gluon distribution function at $R=2 \mathrm{GeV}^{-1}$ compared with the gluon distributions from the CDP model [5], the parametrization model [25], and MSTW08 next-to-next-to-leading order (NNLO) [46]. 

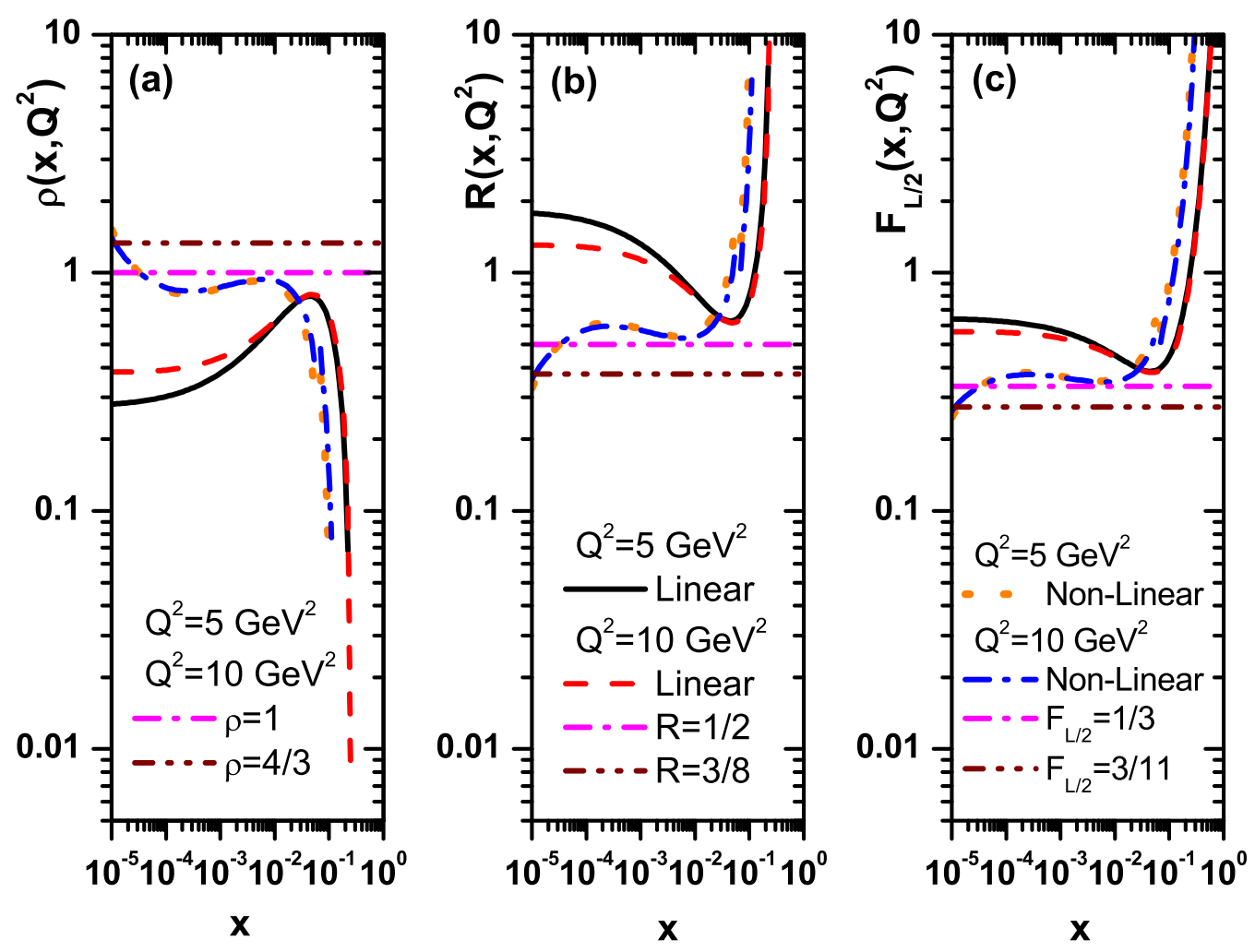

FIG. 2. Results of the parameters (a) $\rho\left(x, Q^{2}\right)$, (b) $R\left(x, Q^{2}\right)$, and (c) $F_{L / 2}\left(x, Q^{2}\right)$ obtained from the linear and nonlinear corrections at fixed $Q^{2}$ values (black-linear and orange-nonlinear: $Q^{2}=5 \mathrm{GeV}^{2}$; red-linear and blue-nonlinear $Q^{2}=10 \mathrm{GeV}^{2}$ ), respectively. The parameters compared with the CDP bounds in (a) $\rho=1$ and $4 / 3$, in (b) $R=1 / 2$ and $3 / 8$, and in (c) $F_{L / 2}=1 / 3$ and $3 / 11$, respectively.

a free parameter as $C_{\mathrm{HT}}=0.12 \pm 0.07 \mathrm{GeV}^{2}$ and in others $[42,43]$ it depends on $x$ as

$$
C_{\mathrm{HT}}(x)=h_{0}\left[h_{2}(x) x^{h_{1}}+\gamma\right] .
$$

In Refs. [42,43] this fit parametrization is obtained from the QCD analysis with the HT corrections included.

Therefore, it is clear from Eqs. (13) and (14) that at low $Q^{2}$, we can add the HT corrections and our solution takes the form

$$
\begin{aligned}
& \sigma_{r}^{\mathrm{NL}+\mathrm{HT}}\left(W^{2}, Q^{2}\right) \\
& \quad=F_{2}^{\mathrm{HT}}\left(W^{2}, Q^{2}\right)\left[1-\frac{y^{2}}{1+(1-y)^{2}} \frac{1}{1+2 \rho^{\mathrm{NL}+\mathrm{HT}}\left(W^{2}, Q^{2}\right)}\right],
\end{aligned}
$$

with

$$
\rho^{\mathrm{NL}+\mathrm{HT}}\left(x, Q^{2}\right)=\frac{27 \pi}{20 \alpha_{s}\left(Q^{2}\right)} \frac{F_{2}^{\mathrm{HT}}\left(x, Q^{2}\right)}{G^{\mathrm{NL}}\left(x, Q^{2}\right)}-\frac{1}{2} .
$$

\section{RESULTS AND DISCUSSIONS}

In this paper, we obtain the nonlinear gluon distribution function solving the GLR-MQ evolution equation for gluon density. The analysis is performed in the ranges of $10^{-5} \leqslant$ $x \leqslant 10^{-2}$ and $1 \leqslant Q^{2} \leqslant 100 \mathrm{GeV}^{2}$. The computed results of the nonlinear gluon distribution function are compared with the CDP model in Kuroda and Schildknecht [5] and the parametrization model [25] in Fig. 1. According to Fig. 7 in Ref. [5], there is considerable agreement with the results from the CETQ Collaboration [44] and Ref. [45]. The nonlinear gluon distribution behavior is comparable with MSTW08 NNLO [46] at $Q^{2}>1 \mathrm{GeV}^{2}$.

In the following, the parameters and bounds with respect to the nonlinear gluon distribution behavior can be examined. With the obtained $\rho$ parameter, we calculate the ratio of structure functions and the reduced cross sections with respect to the nonlinear and higher-twist corrections. These functions are obtained at low- $x$ and low- $Q^{2}$ values by taking an appropriate input parton distribution. In Fig. 2, the parameters $\rho, R$, and $F_{L / 2}$ are obtained with respect to the nonlinear behavior of the gluon distribution function. In the following we have investigated the effect of nonlinearity in our results in the hot-spot point. The value of this parameter is defined to be $R=2 \mathrm{GeV}^{-1}$ in this paper. In Fig. 2 we show that the nonlinear results are much closer to the color dipole bounds than the linear ones. The comparison is for $Q^{2}=5$ and $Q^{2}=10 \mathrm{GeV}^{2}$. The fluctuations corresponding to the parameters (i.e., $\rho, R$, and $F_{L / 2}$ ) in comparison with constant $\mathrm{CDM}$ bounds are due to the parametrization of the PDFs. By adding the effect of the HT corrections on the parameters, we showed that the results have a behavior comparable to the CDM bounds. In Fig. 3, a comparison for $Q^{2}=5 \mathrm{GeV}^{2}$ has been performed between the nonlinear and the nonlinear + higher-twist $(\mathrm{NL}+\mathrm{HT})$ corrections to the parameters. In the following we will apply these corrections (i.e., $\mathrm{NL}+\mathrm{HT}$ ) 

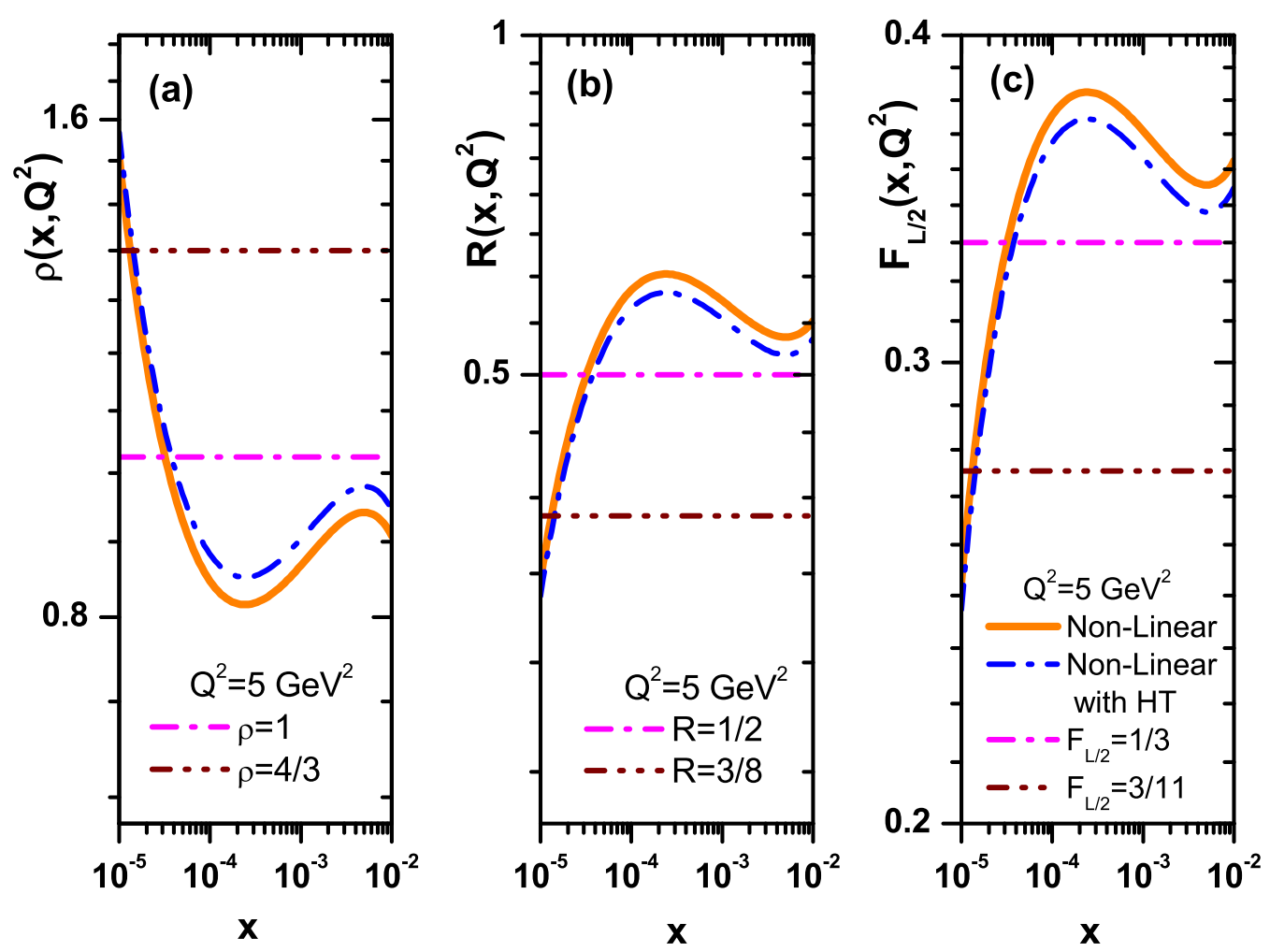

FIG. 3. Comparison of the nonlinear behavior of the parameters (a) $\rho\left(x, Q^{2}\right)$, (b) $R\left(x, Q^{2}\right)$, and (c) $F_{L / 2}\left(x, Q^{2}\right)$ with the higher-twist corrections at $Q^{2}=5 \mathrm{GeV}^{2}$. The parameters compared with the CDP bounds in (a) $\rho=1$ and $4 / 3$, in (b) $R=1 / 2$ and $3 / 8$, and in (c) $F_{L / 2}=1 / 3$ and $3 / 11$, respectively.

to all results. As can be observed in Fig. 4, the ratio of the structure functions are comparable to the $\mathrm{H} 1$ Collaboration data [47] and CDM bounds [3,4,48] not only at high- $Q^{2}$, but

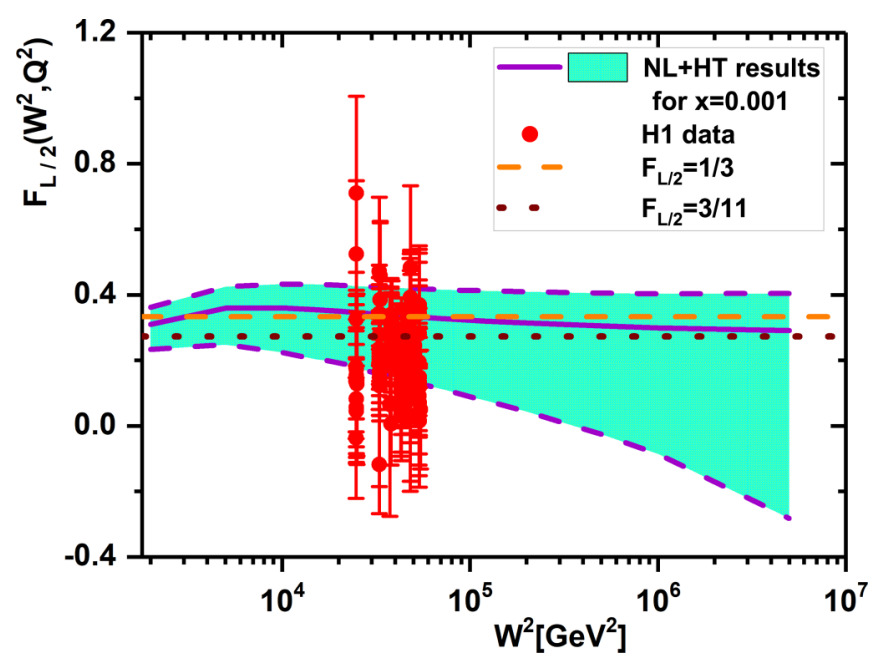

FIG. 4. The ratio of the longitudinal-to-transversal structure functions calculated due to the nonlinear and higher-twist effects at fixed value of the Bjorken variable $x=0.001$. Experimental data are from the $\mathrm{H} 1$ Collaboration as accompanied with total errors [47]. The obtained values compared with the CDP bounds $[3,4,48] F_{L / 2}=1 / 3$ and $3 / 11$. The error bands are due to the effective parameters in the parametrization of $F_{2}\left(x, Q^{2}\right)$ [25] and the HT coefficient errors [36-43]. also at low- $Q^{2}$ values. Indeed, the transition from the linear to nonlinear is performed due to the nonlinear corrections to the gluon distribution function. Compared to other results and

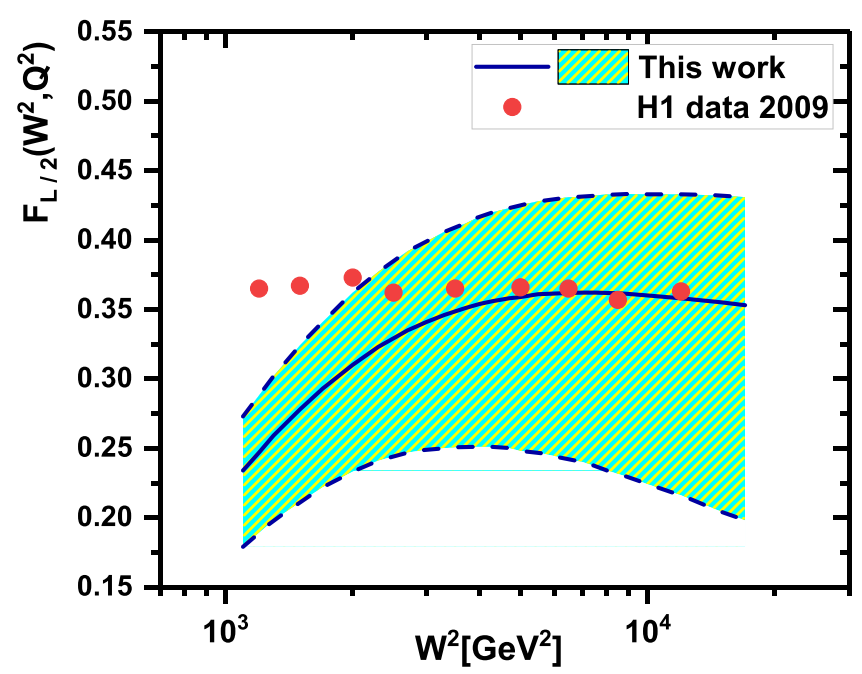

FIG. 5. Continue Fig. 4 in the low- $Q^{2}$ values. The ratio of the longitudinal-to-transversal structure functions calculated due to the nonlinear and higher-twist effects at the fixed value of the Bjorken variable $x=0.001$. Data are from the H1 Collaboration [41] without the total uncertainties at low $Q^{2}$. The error bands are due to the effective parameters in the parametrization of $F_{2}\left(x, Q^{2}\right)$ [25] and the HT coefficient errors [36-40,42,43]. 
TABLE I. The reduced cross-section $\sigma_{r}$ determined based on the nonlinear and higher-twist effects in $Q^{2}$-values 2,5 , and $12 \mathrm{GeV}^{2}$ at $x<0.01$. These results are accompanied with the uncertainties due to the coefficient functions errors [25] and compared with the H1 data [41] as the uncertainties are quoted in percentages relative to $\sigma_{r}$.

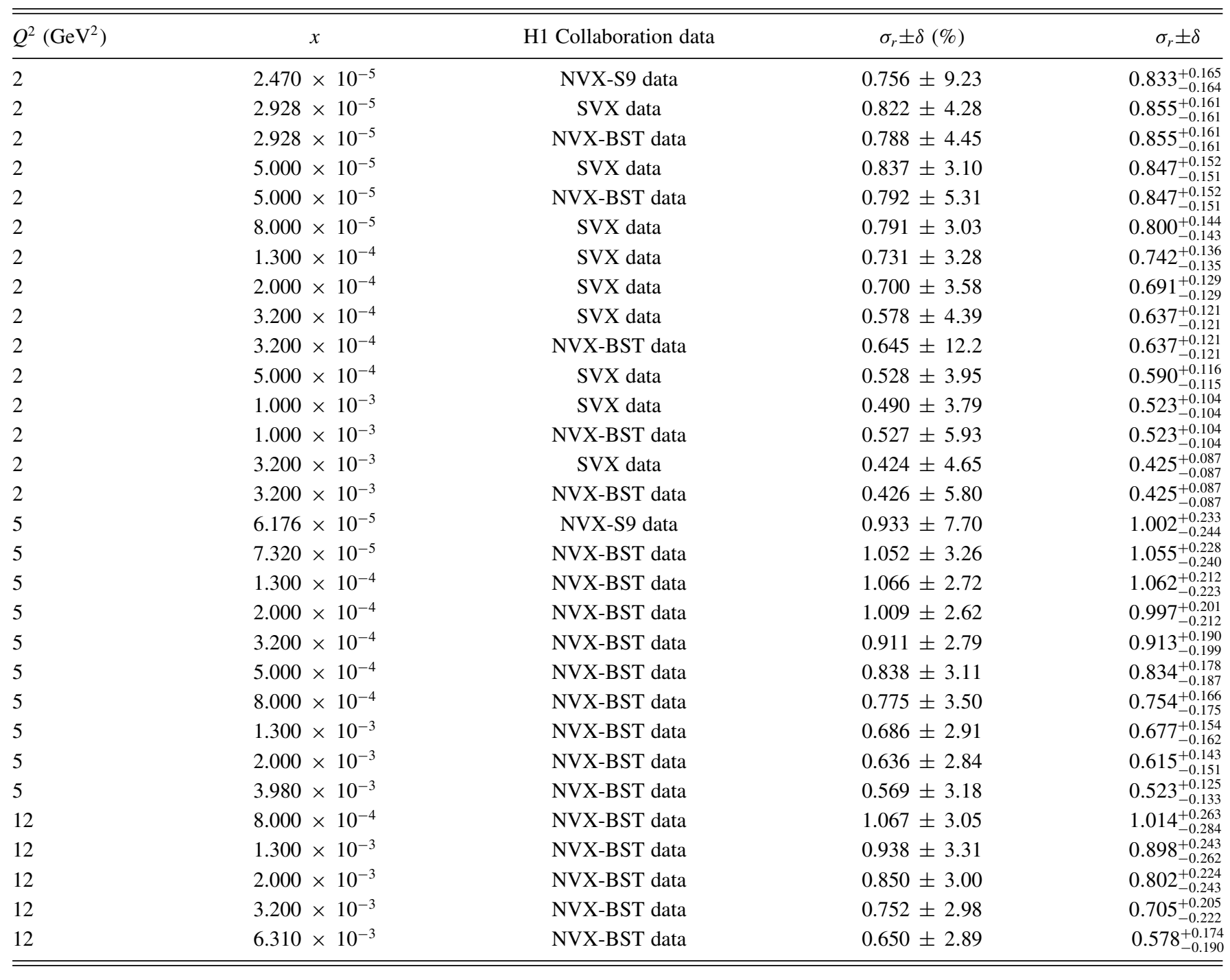

models, we see that the ratio $F_{L} / F_{2}$ is, in fact, comparable to the results of others $[10,49]$ and experimental data. This comparison is very good at low- and high- $Q^{2}$ values, even compared to other models, such as Golec-Biernat-Wüsthoff [10] and Iancu-Itakura-Munier [49] parametrizations. The nonlinear behavior of the ratio of structure functions at low $Q^{2}$ in Fig. 5 is observable in comparison with the H1 Collaboration data [41]. In Fig. 5, data collected in the region of low-momentum transfers $0.2 \mathrm{GeV}^{2} \leqslant Q^{2} \leqslant 12 \mathrm{GeV}^{2}$ and low Bjorken $x, 10^{-6} \lesssim x \lesssim 0.02$ with center-of-mass energy $\sqrt{s}=319 \mathrm{GeV}$. In Ref. [41] the structure functions of $F_{2}$ and $F_{L}$ were collected without the total errors. Tables 17 and 18 in this reference show that $F_{L}^{t h}$ represents the structure function $F_{L}$ used for the center-of-mass energy correction and to calculate the structure function $F_{2}$. Therefore, we compared our results at $x=0.001$ in a wide range of $W^{2}$ between $10^{3}$ and $10^{4} \mathrm{GeV}^{2}$ with the ratio of structure functions (i.e., Ref. [41]) without the total uncertainties in Fig. 5.
In the following we use the NL $+\mathrm{HT}$ behavior of the ratio $F_{L} / F_{2}$ to calculate the reduced cross section. In Ref. [41] the H1 Collaboration reported the DIS cross sections at low $Q^{2}$. The DIS data collected based on the shifted vertex (SVX), nominal vertex-backward silicon tracker (NVX-BST), and NVX-S9 analysis [41]. We use the SVX data at $Q^{2}=2 \mathrm{GeV}^{2}$, the NVX-BST data, the NVX-S9 data at $Q^{2}=5 \mathrm{GeV}^{2}$, and the NVX-BST data at $Q^{2}=12 \mathrm{GeV}^{2}$. The cross-section data due to the $\mathrm{NL}+\mathrm{HT}$ effects at three values of $Q^{2}$ are given in Table I and compared with the $\mathrm{H} 1$ Collaboration data [41] measured from the SVX and NVX data. Here we discuss the $\chi^{2}$ method for comparison according to the number of points at any $Q^{2}$ value. $\chi^{2}$ can be defined as

$$
\chi^{2}=\sum_{i}^{N_{\text {data }}}\left(X_{\text {data }, i}-X_{\text {method }, i}\right)^{2} /\left(\delta X_{i}\right)^{2}
$$


TABLE II. The values of $\chi^{2} / N$ for the computed $\sigma_{r}$ to the H1 Collaboration data [41] in the small- and moderate- $Q^{2}$ regions for $x<0.01$ are determined. Also the number of data points in each case is mentioned.

\begin{tabular}{lccc}
\hline \hline$Q^{2}\left(\mathrm{GeV}^{2}\right)$ & H1 Collaboration data & $N$ & $\chi^{2} / N$ \\
\hline \multicolumn{4}{l}{ NVX-S9 data } \\
2 & NVX-BST data & 15 & 1.696 \\
& SVX data & & \\
& NVX-S9 data & & \\
5 & NVX-BST data & 10 & 1.021 \\
12 & NVX-BST data & 5 & 5.315 \\
\hline \hline
\end{tabular}

where $i$ runs all the data points $\delta X_{i}$ can be the total experimental uncertainties. The $\chi^{2} / N_{\text {data }}$ can quantify the agreement between the data and our predictions. The $\chi^{2} / N_{\text {data }}$ computed at different values of $Q^{2}$ is in Table II.

The results are plotted in Fig. 6. In this figure, together with the H1 Collaboration data [41], we plot the $x$ dependence of the reduced cross-section $\sigma_{r}\left(x, Q^{2}\right)$ computed with respect to the nonlinear and higher-twist effects for fixed values of $Q^{2}$. The error bands are in accordance with the statistical errors of the parametrization of $F_{2}$, and the errors bars are quoted in percentages relative to $\sigma_{r}$. The agreement of these results with the H1 Collaboration data are excellent for $x<0.01$. We also compare these results with the HERA data [50] (which combines H1 Collaboration and ZEUS Collaboration data) in Fig. 6 at $Q^{2}$ values of 2 and $12 \mathrm{GeV}^{2}$. This includes data taken with proton beam energies of $E_{p}=920 \mathrm{GeV}$ corresponding to the center-of-mass energy $\sqrt{s}=318 \mathrm{GeV}$. Therefore, the results of the current paper in the region of smallest $x$ and $Q^{2}$ studies can confirm the nonlinear corrections to the small- $x$ gluon distributions for transition.

\section{SUMMARY}

In conclusion, we have studied the effects of adding the nonlinear corrections to the gluon density for transition from the linear to the nonlinear regions. We use the parametrization of $F_{2}\left(x, Q^{2}\right)$ as a baseline. This analysis is also enriched with the HT contributions to the proton structure function at small values of $Q^{2}$. The nonlinear and higher-twist corrections to the ratio of structure functions and to the reduced cross sections are considered. Comparing these parameters with the CDM bounds indicate that the NL + HT effects are enriched by the behavior at low $Q^{2}$. The transition of the ratio $F_{L / 2}$ from the linear to the nonlinear behavior is considered and shows that it is in good agreement with the CDM bounds not only at high- $Q^{2}$, but also at low- $Q^{2}$ values. Comparison of the reduced cross sections with respect to the

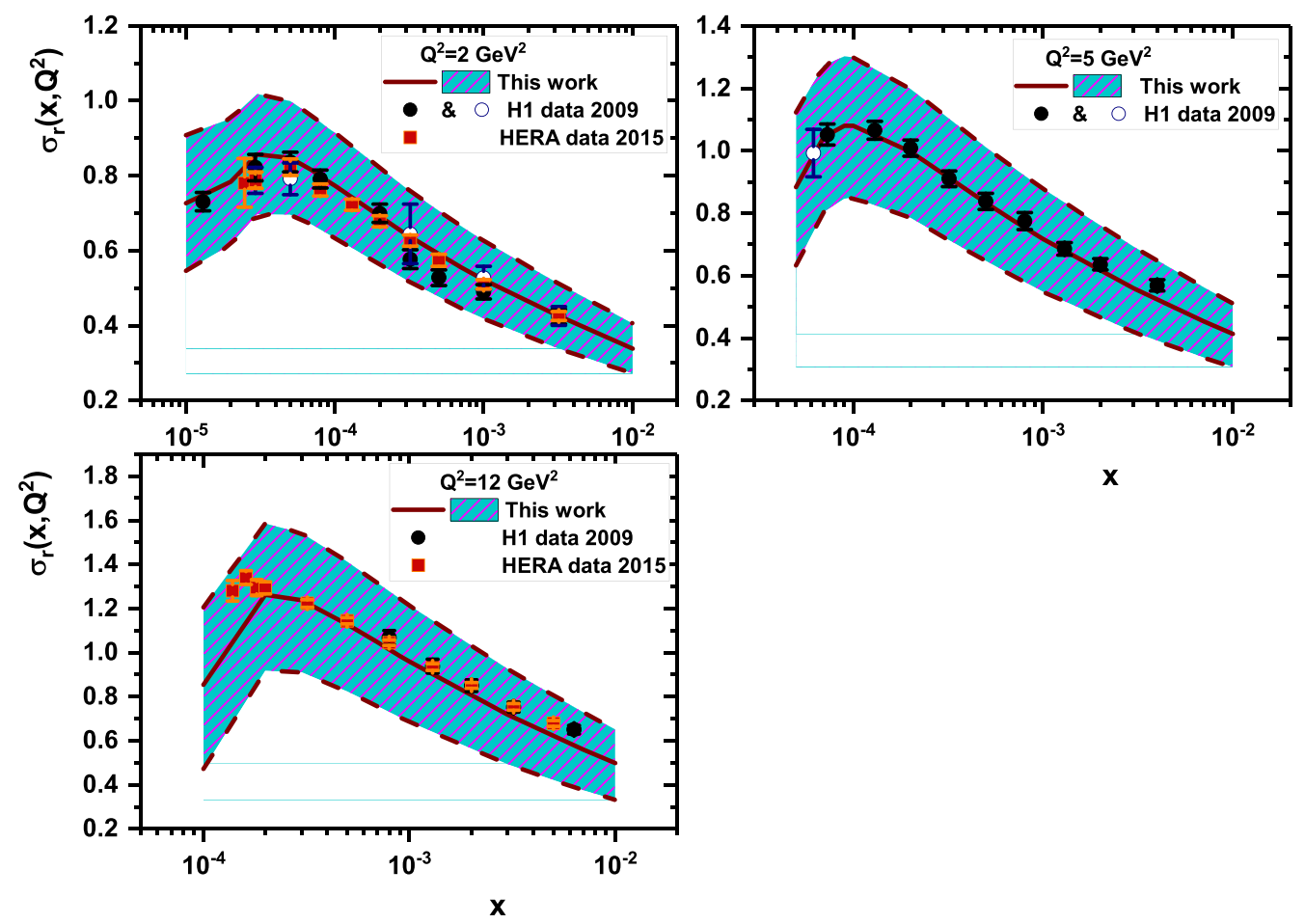

FIG. 6. Reduced cross-section $\sigma_{r}^{\mathrm{NL}+\mathrm{HT}}$ from the nonlinear behavior of the gluon distribution and the higher-twist corrections to the proton structure function at low $x$ and low $Q^{2}$ compared to the reduced cross-section $\sigma_{r}$ from the combined low- $Q^{2}$ data [41] and HERA combined data [50]. H1 Collaboration data accompanied with total errors. H1 Collaboration data represented for $Q^{2}=2 \mathrm{GeV}^{2}$ as the closed circles are SVX data, and the open circles are NVX-BST data for $Q^{2}=5 \mathrm{GeV}^{2}$, the closed circles are NVX-BST data, and the open circles are NVX-S9 data, and for $Q^{2}=12 \mathrm{GeV}^{2}$ the closed circles are NVX-BST data [41]. The error bands are due to the effective parameters in the parametrization of $F_{2}\left(x, Q^{2}\right)$ [25] and the HT coefficient errors [36-40,42,43]. This comparison with HERA combined data [50] at $Q^{2}=2$ and 12 GeV ${ }^{2}$ is defined. 
nonlinear and higher-twist corrections with HERA data at low and moderate $Q^{2}$ values shows that this transition has been performed with good accuracy in comparison with the HERA data.

\section{ACKNOWLEDGMENTS}

We are grateful to the Razi University for financial support of this project. G.R.B. was especially grateful to D. Schildknecht for carefully reading the paper and fruitful discussions.
[1] J. J. Sakurai and D. Schildknecht, Phys. Lett. B 40, 121 (1972); B. Gorczyca and D. Schildknecht, ibid. 47, 71 (1973).

[2] N. N. Nikolaev and B. G. Zakharov, Z. Phys. C: Part Fields 49, 607 (1991); 53, 331 (1992); A. H. Mueller, Nucl. Phys. B 415, 373 (1994); K. Golec-Biernat and M. Wüsthoff, Phys. Rev. D 59, 014017 (1998); H. Kowalski, L. Motyka, and G. Watt, ibid. 74, 074016 (2006); B. Sambasivam, T. Toll, and T. Ullrich, Phys. Lett. B 803, 135277 (2020); G. M. Peccini, F. Kopp, M. V. T. Machado, and D. A. Fagundes, Phys. Rev. D 101, 074042 (2020).

[3] M. Kuroda and D. Schildknecht, Phys. Lett. B 618, 84 (2005); Phys. Rev. D 96, 094013 (2017); D. Schildknecht, B. Surrow, and M. Tentynkov, Eur. Phys. J. C 20, 77 (2001); M. Kuroda and D. Schildknecht, Int. J. Mod. Phys. A 31, 1650157 (2016).

[4] D. Schildknecht, Nucl. Phys. B, Proc. Suppl. 146, 211 (2005); Nucl. Phys. B Proc. Suppl. 222-224, 108 (2012); F. Schrempp and A. Utermann, Acta Phys. Pol. B 33, 3633 (2002).

[5] M. Kuroda and D. Schildknecht, Phys. Lett. B 670, 129 (2008); Phys. Rev. D 85, 094001 (2012).

[6] D. Boer et al., arXiv:1108.1713.

[7] J L Abelleira Fernandez et al. (LHeC Study Group Collaboration), J. Phys. G 39, 075001 (2012); A. Abada et al. (FCC Study Group Collaboration), Eur. Phys. J. C 79, 474 (2019).

[8] P. Agostini et al. (LHeC Collaboration and FCC-he Study Group), arXiv:2007.14491.

[9] H. Kowalski and D. Teaney, Phys. Rev. D 68, 114005 (2003); G. Watt and H. Kowalski, ibid. 78, 014016 (2008).

[10] J. Bartels, K. Golec-Biernat, and H. Kowalski, Phys. Rev. D 66, 014001 (2002); K. Golec-Biernat and M. Wüsthoff, ibid. 60, 114023 (1999); K. Golec-Biernat and S. Sapeta, J. High Energy Phys. 03 (2018) 102.

[11] M. Klein, arXiv:1802.04317; Annalen Phys. 528, 138 (2016); N. Armesto, P. R. Newman, W. Slomiński, and A. M. Staśto, Phys. Rev. D 100, 074022 (2019).

[12] L. V. Gribov, E. M. Levin, and M. G. Ryskin, Phys. Rep. 100, 1 (1983); A. H. Mueller and J. w. Qiu, Nucl. Phys. B 268, 427 (1986).

[13] M. R. Pelicer et al., Eur. Phys. J. C 79, 9 (2019).

[14] A. D. Martin, R. G. Roberts, W. J. Stirling, and R. S. Thorne, Eur. Phys. J. C 23, 73 (2002); Phys. Lett. B 531, 216 (2002).

[15] P. M. Nadolsky et al., Phys. Rev. D 78, 013004 (2008).

[16] W. Zhu, J. Ruan, J. Yang, and Z. Shen, Phys. Rev. D 68, 094015 (2003).

[17] I. Balitsky, Nucl. Phys. B 463, 99 (1996); Y. V. Kovchegov, Phys. Rev. D 60, 034008 (1999).

[18] J. Jalilian-Marian, A. Kovner, A. Leonidov, and H. Weigert, Nucl. Phys. B 504, 415 (1997); Phys. Rev. D 59, 014014 (1998); E. Iancu, A. Leonidov, and L. D. McLerran, Nucl. Phys. A 692, 583 (2001); E. Ferreiro, E. Iancu, A. Leonidov, and L. D. McLerran, ibid. 703, 489 (2002).

[19] A. H. Rezaeian, M. Siddikov, M. Van de Klundert, and R. Venugopalan, Phys. Rev. D 87, 034002 (2013).

[20] I. I. Balitsky and A. V. Belitsky, Nucl. Phys. B 629, 290 (2002).
[21] V. Barone et al., Phys. Lett. B 326, 161 (1994).

[22] M. Sadzikowski, L. Motyka, and W. Slominski, arXiv:1206.1732.

[23] M. B. Gay Ducati, E. G. de Oliveira and J. T. de Santana Amaral, Eur. Phys. J. C 72, 2196 (2012).

[24] I. P. Ivanov et al., in XXXII International Symposium on Multiparticle Dynamics, Alushta, Ukraine, 2003, edited by A. Sissakian, G. Kozlov, and E. Kolganova (World Scientific, Singapore, 2003), pp. 169-176; K. Tywoniuk, J. Phys.: Conf. Ser. 270, 012054 (2011).

[25] M. M. Block, L. Durand and P. Ha, Phys. Rev. D 89, 094027 (2014).

[26] G. R. Boroun, arXiv:2102.04867.

[27] M. Devee and J. K. Sarma, Eur. Phys. J. C 74, 2751 (2014); Nucl. Phys. B 885, 571 (2014); P. Phukan, M. Lalung, and J. K. Sarma, Nucl. Phys. A 968, 275 (2017); M. Lalung, P. Phukan, and J. K. Sarma, ibid. 992, 121615 (2019); M. Devee, arXiv:1808.00899; G. C. Penedo and W. K. Sauter, arXiv:1804.10659.

[28] G. R. Boroun, Eur. Phys. J. A 42, 251 (2009).

[29] G. R. Boroun, Phys. Rev. C 97, 015206 (2018).

[30] B. Rezaei and G. R. Boroun, Phys. Rev. C 101, 045202 (2020).

[31] B. Rezaei and G. R. Boroun, Eur. Phys. J. A 56, 262 (2020).

[32] G. R. Boroun and S. Zarrin, Eur. Phys. J. Plus 128, 119 (2013).

[33] G. R. Boroun and B. Rezaei, Nucl. Phys. A 1006, 122062 (2021).

[34] K. Prytz, Eur. Phys. J. C 22, 317 (2001); K. J. Eskola et al., Nucl. Phys B 660, 211 (2003); M. A. Kimber, J. Kwiecinski and A. D. Martin, Phys. Lett. B 508, 58 (2001).

[35] A. D. Martin, W. J. Stirling, and R. G. Roberts, Phys. Rev. D 47, 867 (1993); J. Kwiecinski, A. D. Martin, and P. J. Sutton, ibid. 44, 2640 (1991); A. J. Askew, J. Kwiecinski, A. D. Martin, and P. J. Sutton, ibid. 47, 3775 (1993).

[36] B. Badelek et al., J. Phys. G: Nucl. Part. Phys. 22, 815 (1996); S. Catani and F. Hautmann, Nucl. Phys. B 427, 475 (1994); J. Blümlein and H. Böttcher, arXiv:0807.0248.

[37] A. M. Cooper-Sarkar, I. Abt, B. Foster, M. Wing, V. Myronenko, and K. Wichmann, arXiv:1605.08577.

[38] I. Abt, A. M. Cooper-Sarkar, B. Foster, V. Myronenko, K. Wichmann, and M. Wing, Phys. Rev. D 94, 034032 (2016).

[39] G. R. Boroun and B. Rezaei, Phys. Lett. B 816, 136274 (2021).

[40] G. R. Boroun and B. Rezaei, Nucl. Phys. A 990, 244 (2019).

[41] F. D. Aaron et al. (H1 Collaboration), Eur. Phys. J. C 63, 625 (2009).

[42] H. Khanpour, A. Mirjalili, and S. A. Tehrani, Phys. Rev. C 95, 035201 (2017).

[43] H. Khanpour, Phys. Rev. D 99, 054007 (2019).

[44] J. Pumplin et al. (CTEQ Collaboration), J. High Energy Phys. 07 (2002) 012.

[45] A. D. Martin et al., Eur. Phys. J. C 18, 117 (2000). 
[46] A. D. Martin et al., Eur. Phys. J. C 63, 189 (2009).

[47] V. Andreev et al. (H1 Collaboration), Eur. Phys. J. C 74, 2814 (2014).

[48] C. Ewerz, A. von Manteuffel, and O. Nachtmann, J. High Energy Phys. 03 (2010) 102; D. Britzger, C. Ewerz, S. Glazov, O. Nachtmann, and S. Schmitt, Phys. Rev. D 100, 114007 (2019); C. Ewerz, A. von Manteuffel, and O. Nachtmann, ibid.
77, 074022 (2008); C. Ewerz and O. Nachtmann, Phys. Lett. B 648, 279 (2007).

[49] E. Iancu, K. Itakura, and S. Munier, Phys. Lett. B 590, 199 (2004); M. Niedziela and M. Praszalowicz, Acta Phys. Pol., B 46, 2018 (2015).

[50] H. Abramowicz et al. (H1 Collaboration and ZEUS Collaboration), Eur. Phys. J. C 75, 580 (2015). 